\title{
Neanderthal genome sees first light
}

Two research teams publish the first detailed glimpse of a Neanderthal genome this week, starting the race to unravel the genetic structure of modern man's closest relative. Articles in Nature ${ }^{1}$ and Science $^{2}$ offer complementary yet contrasting views of nuclear DNA extracted from the same bone of a Neanderthal male who lived 38,000 years ago, found in a Croatian cave.

Both groups, which collaborated in part on their projects, say they need to sequence far more of the Neanderthal's DNA to answer key questions. But for now, the studies find roughly similar timescales for when Neanderthals and modern humans, Homosapiens, diverged from each other in the course of evolution.

Given recent advances in DNA sequencing, a full Neanderthal genome could be sequenced within two years, the Nature authors say. ${ }^{\alpha}$ This is the golden age of ancient DNA work," says first author Richard Green, a postdoctoral researcher at the Max Planck Institute for Evolutionary Anthropology in Leipzig, Germany.

Neanderthals and $H$. sapiens roamed the same regions of Europe and western Asia until the Neanderthals went extinct 30,000 years ago. Both research groups now have genetic estimates for when the two species shared a most recent common ancestor.

In the Science article, team leader Edward Rubin places that event at roughly 706,000 years ago. In Nature, the Max Planck group - headed by palaeogeneticist Svante Päăbo - write that human and Neanderthal sequences diverged roughly 516,000 years ago. Within the large margins of error typical of such analyses, these conclusions could turn out to be the same. The Nature paper also takes a stab at estimating the size of the ancestral
"The gene studies may also shed light on a long-contested idea: that Neanderthals and $H$. sapiens may have interbred."

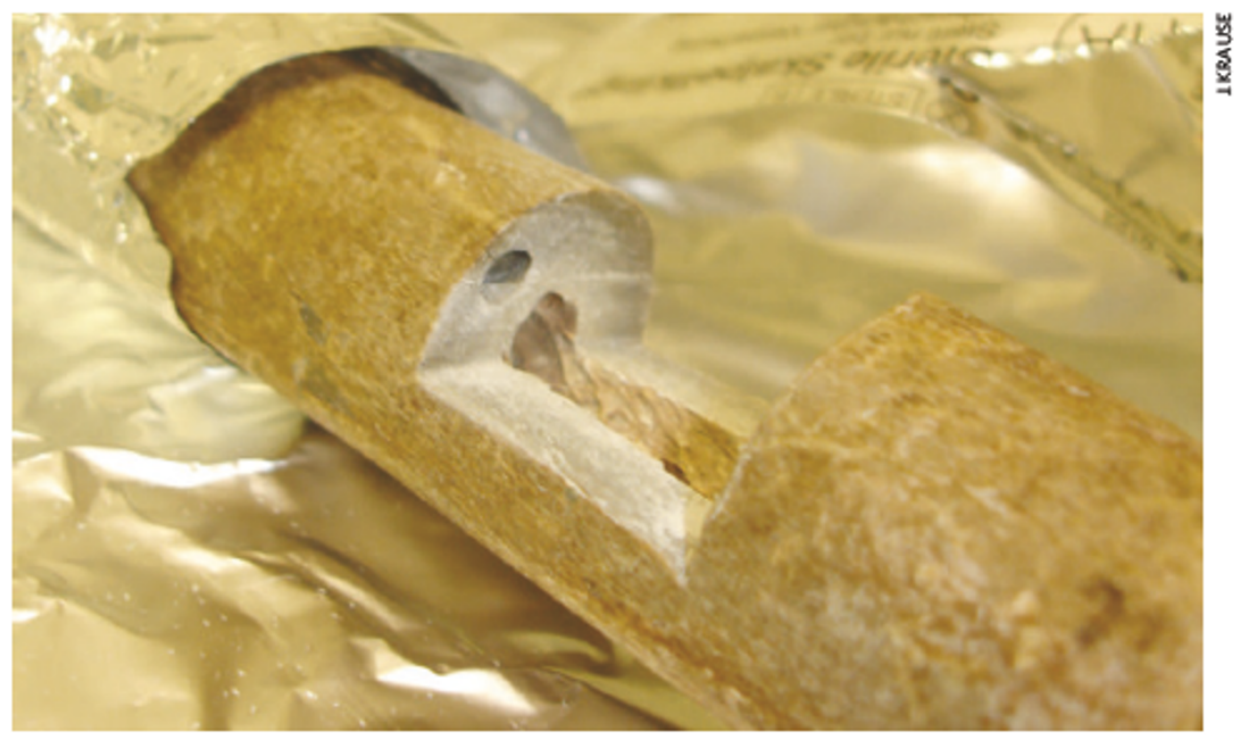

Sequencing of DNA from ancient bone might result in a full Neander thal genome within two years.

ing may have taken place on a limited scale, says Päabo. "One possibility is gene flow from modern human to Neanderthal," he says. But the data could also be explained if the studied Neanderthal sample was contaminated with modern human DNA. "We have contamination under control," Pääbo says.

As for the other group, "we don't see any evidence of interbreeding", says Rubin, director of the Joint Genome Institute (JGI) in Walnut Creek, California. "But we could have missed it because we don't have enough Neanderthal sequence yet."

Rubin's group reports analysis of about 65,000 base pairs. The Nature article reports about 1 million. The genomes of modern humans and Neanderthals each have about 3 billion base pairs. "These studies show you population that gave rise to humans and Nean- can do large-scale sequencing of Neanderthal derthals: roughly 3,000 individuals.

The gene studies may also shed light on a long-contested idea in palaeoanthropology: that Neanderthals and H. sapiens may have interbred. Last week, another research team proposed that modern humans acquired a version of the microcephalin gene - which regulates brain size - through interbreeding with another Homo species that has since gone extinct, possibly the Neanderthals ${ }^{3}$. Fossils - such as a 30,000-year-old skull from a Romanian cave ${ }^{4}$ - have also been put forward as evidence of hybridization between the two species.

The latest data suggest that some interbreed- can do large-scale sequencing of Neanderthal gist at the University of Southern California in Los Angeles who was not involved in either study. ${ }^{\text {TThe }}$ are some unresolved issues, ${ }^{n}$ he says. "In the end, the questions will be answered to everyone's satisfaction."

Both groups agree that less than $0.5 \%$ of the two species' genomes are different. But the teams largely used different sequencing methods. The JGI team opted for a traditional bacterial, or Sanger, method to replicate Neanderthal DNA. The Max Planck team used a newer, and faster, sequencing system ${ }^{5}$.

Both groups are pumping new sequences into publicly accessible databases. Nearly 5 million base pairs are already available. By spring, Paăbo expects to have about $1 \%$ of the Neanderthal genome completed.

Rubin is now developing methods to target specific genes in the regions of the genome that are different in Neanderthals and modern humans. This approach, he says, allows one "to reach in and fish out DNA for analysis for different traits".

One of the first fishing trips will be for a gene called FOXP2, which in $H$. sapiens is linked to speech and language. Many animals have the gene, but the human version has distinct differences from that of the chimpanzee.

Such genetic excursions will need more Neanderthal individuals for a truly representative picture of the differences between the species. Palaeoanthropologists and geneticists are now teaming up to acquire more bone, from more caves.

Eventually, genetics and palaeoanthropology will intertwine, predicts James Noonan, a JGI postdoc and first author on the Science article. "Howbiological features are encoded in the genome, he says, "will be correlated with what is seen in specimens."

\section{RexDalton}

1. Green, R. E et al. Nature 444, 330-336(2006).

2. Noonan, 1 . P.et al. Science 314, 1113-1118 (2006)

3. Evans, P. D.etal. Proc. Natl Acad. Sd. USA doci101073/ pnas0606966103(2006)

4. Soficaru A. Dobo, A.\& Trinkaus, E. Proc Natl Acod. Sci. USA dok:101073/pnas.0608443103(2006)

5. Margulies, M. etal. Nature $437,376-380$ (2005).

6. Enard, W et al. Nature 418, 869-872(2002) 